\title{
Can we tell how climate will change in the future?
}

\section{Frank Oldfield}

Working out how climate will change in the future depends on the use of computer-based models. The latest models of future climate change tell us that during your lifetime, global warming and the weather extremes linked to it will pose major challenges for life on Earth unless urgent action is taken now. How reliable are these models and the projections of future climate that they create? Producing them requires a rather new kind of science that is often criticized by those who don't believe in man-made climate change, so it is important to learn more about how climate models, especially those used to project future global warming, are tested for their reliability.

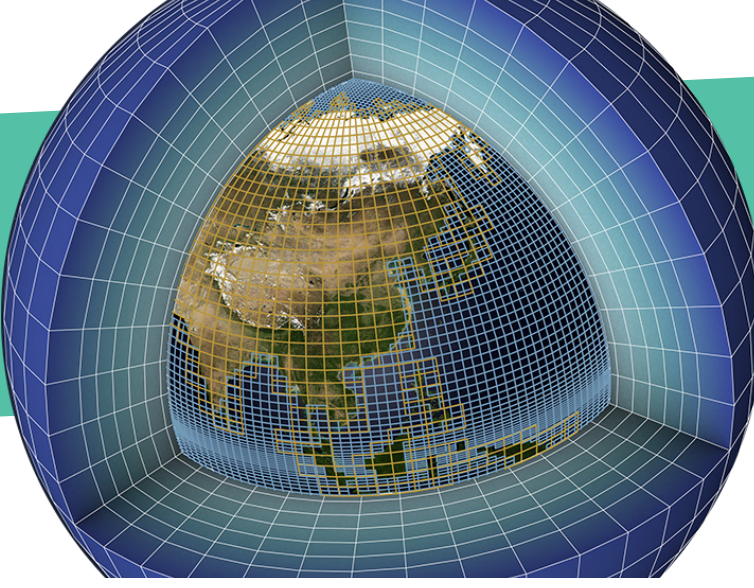

Image credit: IPSL, CEA

A key test is whether they can predict the climate during a period for which we already know what the climate was like: the past! Unlike in modern times, earlier climatic changes were driven by natural processes such as changes in the energy received from the sun, natural variations in atmospheric greenhouse gases such as $\mathrm{CO}_{2}$ and methane, and volcanic activity. Over the last few decades, changes arising from human activities must also be included, mainly increases in atmospheric greenhouse gases and notably $\mathrm{CO}_{2}$ arising largely from the use of fossil fuels. Only if the results produced by the models for the past compare well with the actual record of past climate changes can we rely on the models to give reliable projections for the future. The model-based results are usually referred to as simulations.
HOW WILL CLIMATE CHANGE THE FUTURE?

(2) NOCRYSTAL $\rightarrow$ BALL BUT acrianiachiones

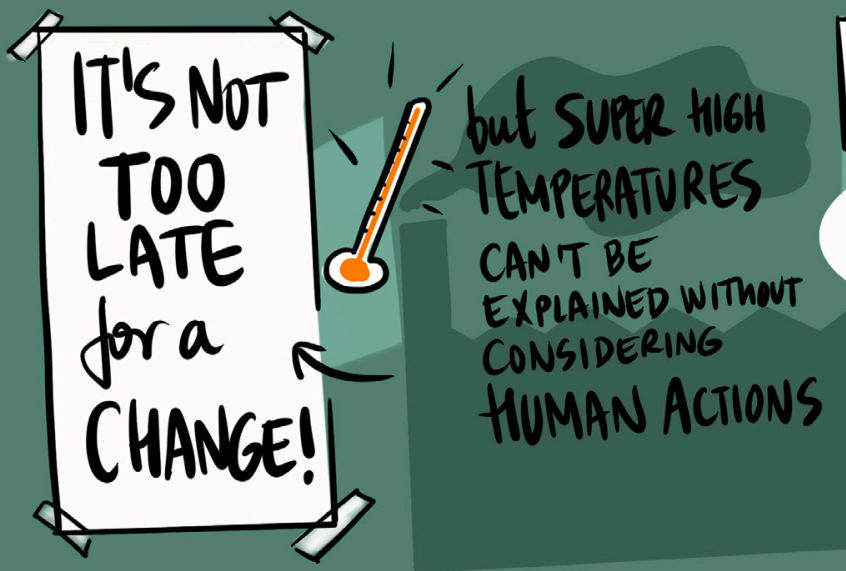

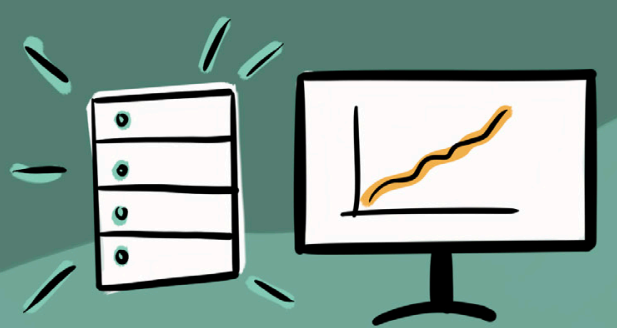

FAMADITER-BASED MODELS USed to make PREDICTIONS =
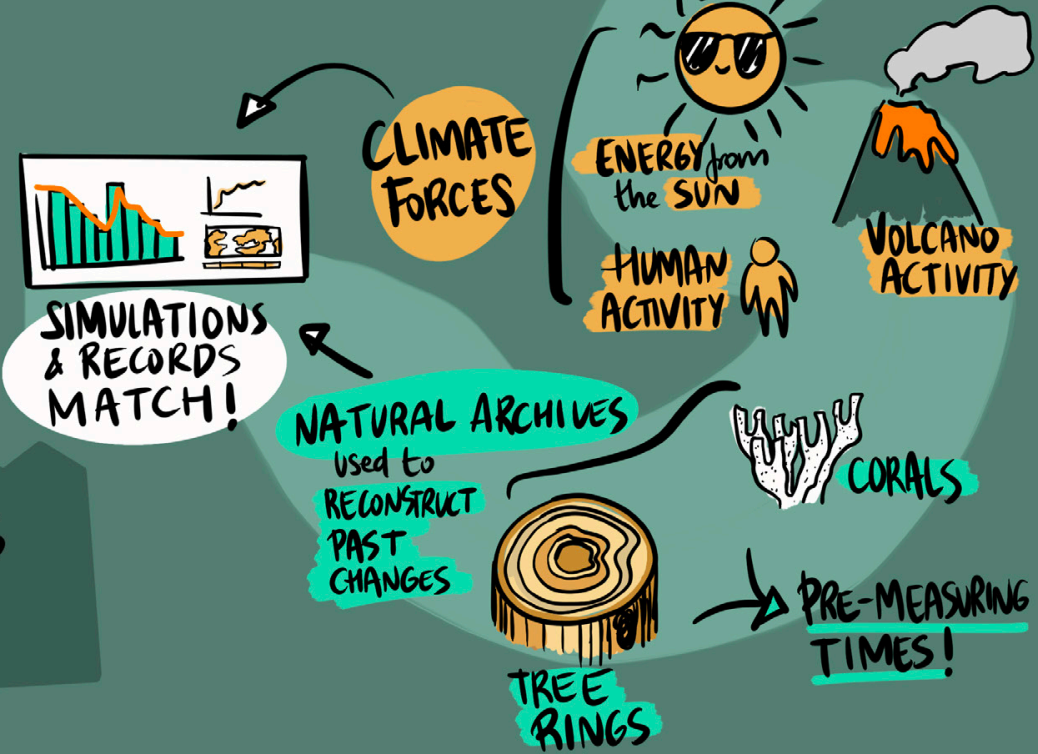
Wait but... DO THEY $>$ WORK? $\sqrt{5}$ TEST TUE PAST FIRST 
We know mainly from meteorological and written records how climate has changed over the last 170 years or so. Models can therefore be tested by comparing the simulations for this period with the actual record of climate change.

But climate has varied on much longer timescales, so it is also important to be able to make similar comparisons for a longer period in the past. For pre-measurement times, past global temperatures have to be reconstructed from other lines of evidence, for example tree rings, sediments, and corals. We may think of these as natural archives of information about the climate. First, we need to work out, through a process of calibration, how well they have recorded past climate change.

Take tree rings as one example. Accurately dated features like annual ring width for a group of trees can be established for each year that the trees grew. The sequence obtained can then be compared with the temperature records spanning the same period from nearby weather stations. By comparing the sequence of tree-ring measurements with the sequence of temperature values, the tree-ring record can be translated into past temperature changes. Then, by measuring ring widths in dated older wood, beyond the timespan of direct observations, the values derived from the calibration can be used to reconstruct a longer record of annual temperature changes. The next step is to combine records from long-lived trees with those from fossil wood. These can then provide temperature records spanning many centuries. The methods for calibrating other natural archives may be different, but the goal remains the same, to derive records of past climate change for the period before the 1850 s.
Now we come to the key question: how well do the model-based simulations of past temperature compare with the records derived from measurements and from natural archive-based reconstructions? Figure 1 shows a reconstruction of global temperatures for the period 1200 to $1850 \mathrm{CE}$ compared with the results from a range of the most recent computer-based global temperature simulations for the same period. The graph shows that there is good agreement between the model-based simulations and the grey envelope of natural archive-based reconstructions. So far so good, but what about the recent past, during which time human activities have affected the climate?

Figure 2 shows the record for the most recent period, the one during which the key question of human-induced global warming arises. The graph provides a comparison between the past changes in global temperature over the last 170 years and the calculated effects of the various processes, both natural and human-induced, that are used to generate computer-based climate models. Until the middle of the 20th century, the changes in global temperature mainly reflect the influence of natural processes, but since then those natural processes on their own fail to generate changes that match the actual, rapid temperature increase recorded

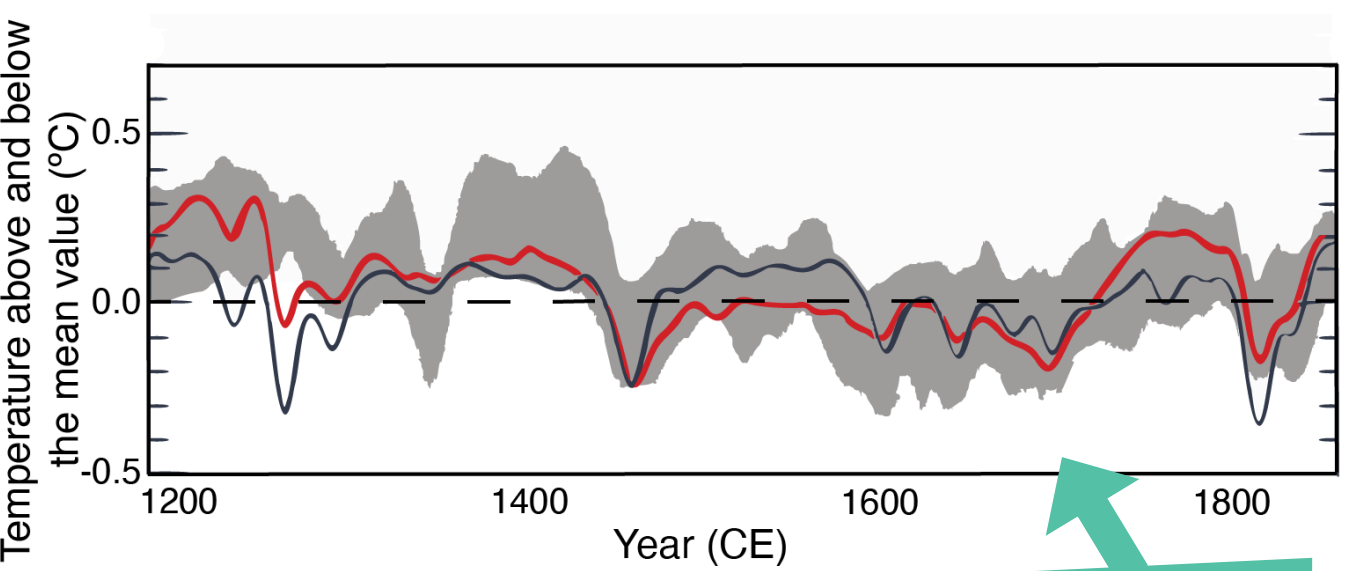
Figure 1: The graph compares computer-simulated results with natural archive-based reconstructions of global temperatur for multiple model1850 CE. The red and blue lines plot mean values for multiple mariations in based simulations. The red line uses maximum values for values. The grey shing the sun's influence, the blue line uses mes by several independent research spans the range of reconstructed values by selions above and below groups. The temperature scale shows variations abo 1500-1850 (dashed line). reconstructed mean temperature for the per in the Fifth Assessment Report The graph is based on Figure 5.8 shown
of the Intergovernmental Panel on Climate Change. 


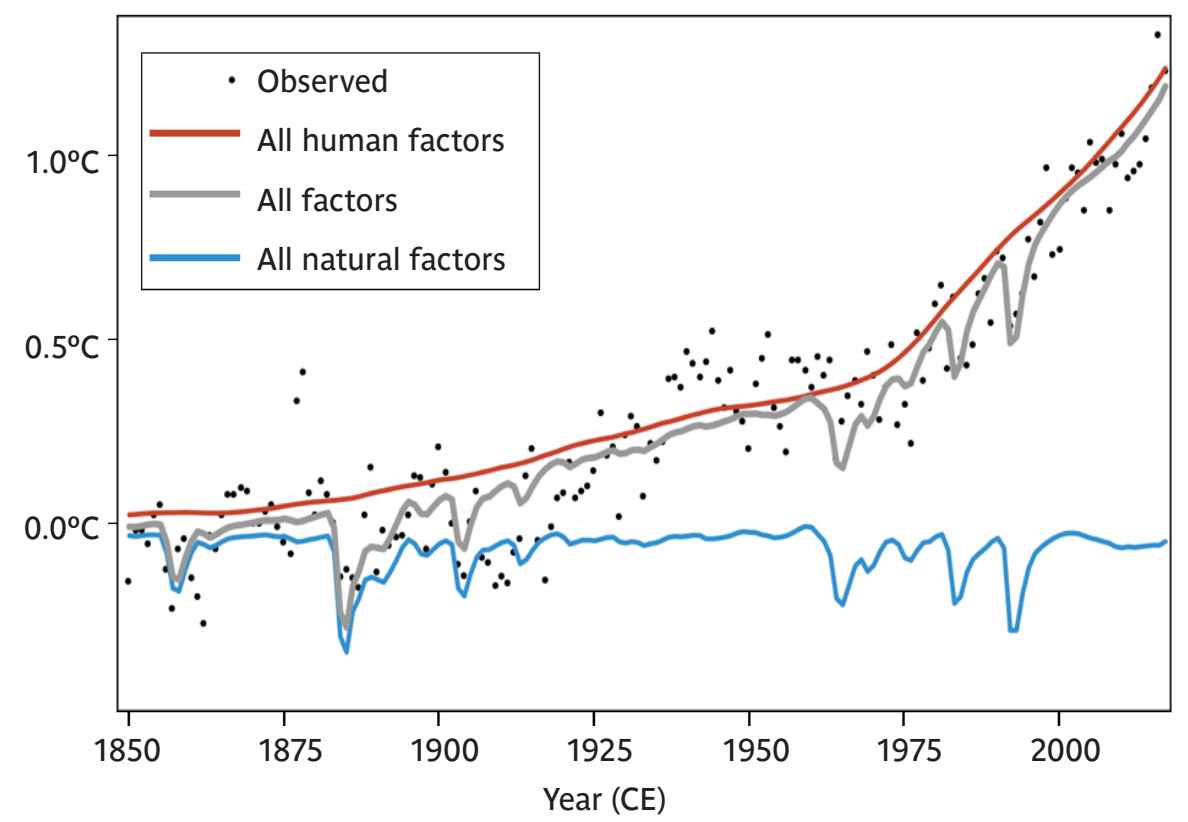

Figure 2: The graph compares computer-simulated results with the actual observed record of global temperature for the period since 1850 CE. The black dots represent annual values and the lines plot the results of alternative simulations, both with and without the impact of human activities. Adapted from Global Warming Index.

by recent measurements. Only by factoring in the rise in greenhouse gas concentrations resulting largely from fossil

fuel use is it possible to account for this increase. From these graphs, we see that models can reproduce the past record of climate change extremely well; moreover, the second graph confirms that the main cause of the recent increase in temperature is human activity. These results strengthen confidence in the projections of future global temperature

\section{Alternative simulated global temperature trends to 2100}

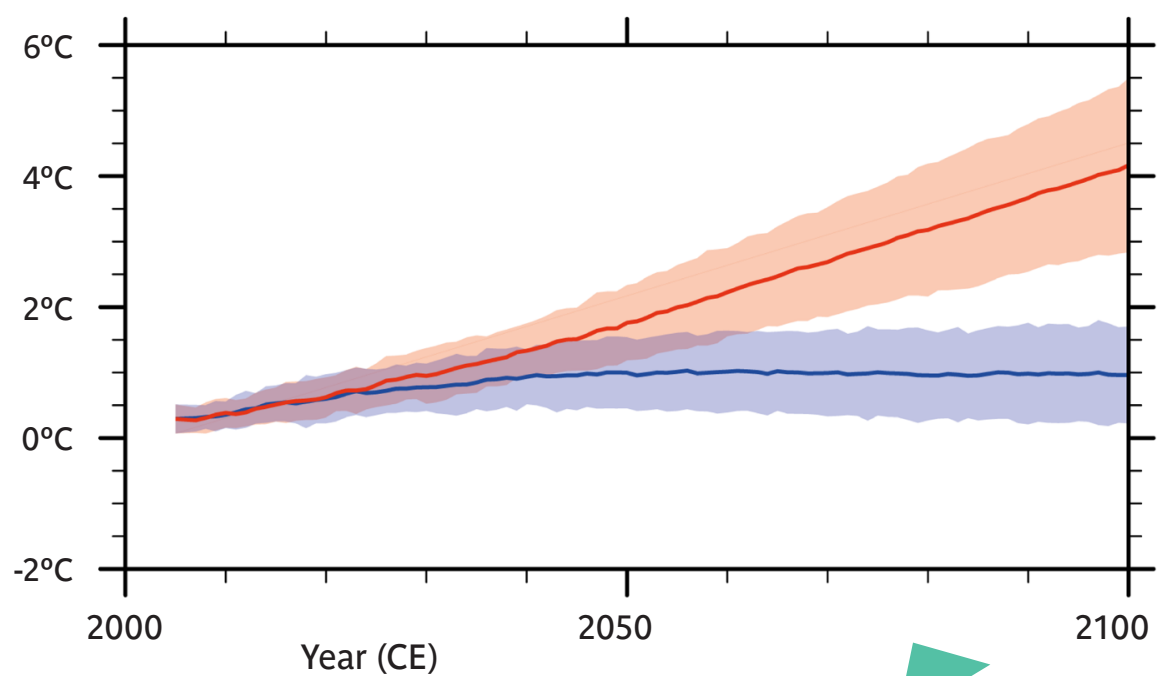

Figure 3: The graph shows one of many attempts to highlight the likely impact of alternative human responses on the future continued of global temperature changes. The red line line assumes urgent and high greenhouse gas emissions; the bling shows the uncertainty optimum reduction and control. Shapted from Figure SPM.6. attached to each projection. that models provide. How can anyone doubt their value as a basis for action?

Enough about the science used to validate future global temperature projections - the actual course of climate change during your lifetime and beyond will depend, above all, on human behavior. Projecting the likely impact on climate change of different combinations of human activities calls for models that go beyond the strictly scientific. They inevitably include additional uncertainties. Figure 3 is one of many attempts to show the crucial importance of human behavior. The two lines are shaded to indicate the level of uncertainty for each projection. If there is an urgent, optimum reduction in emissions we would expect to follow the blue line. If there is continued, rapid growth in atmospheric concentrations of greenhouse gases the climate models suggest that we will follow the red line. The future therefore depends on human policy and responses.

Look at the graph and ask yourself: what needs to happen to bring further increases in greenhouse gases to levels that minimize future environmental damage and human hardship? (3) 